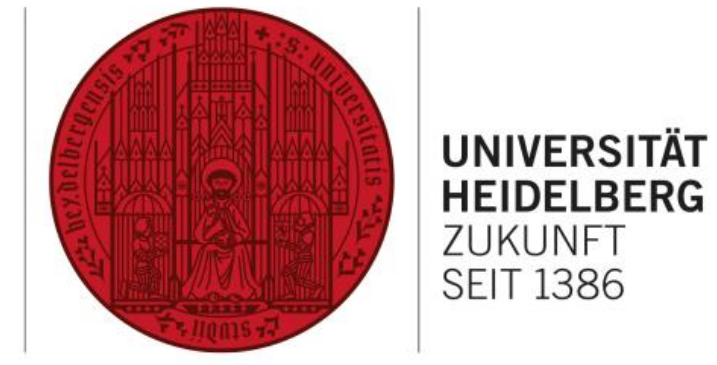

Zusammenfassung der Dissertation mit dem Titel

\title{
„Die Notwendigkeit der rechtlichen Harmonisierung der Lebendorganspende auf europäischer und internationaler Ebene $^{66}$
}

Dissertation vorgelegt von Katharina Ludwig

Erstgutachter: Prof. Dr. Gerhard Dannecker

Zweitgutachter: Prof. Dr. Dr. h.c. Thomas Hillenkamp

Institut für deutsches, europäisches und internationales Strafrecht und Strafprozessrecht 
Die vorliegende Dissertation befasst sich mit dem Problem der rechtlichen Harmonisierung der Lebendorganspende im Mehr-Ebenen-System.

Anlass der Untersuchung war die geplante Harmonisierung der Organspende durch einen Aktionsplan im Bereich der Organspende und -transplantation der europäischen Kommission aus dem Jahr 2008 der mit einem Richtlinienvorschlag kombiniert und anschließend vom Europäischen Parlament im Jahr 2010 angenommen wurde. Der Aktionsplan und die Richtlinie der Kommission betonten, dass der Organmangel in Europa ein zunehmend drängendes Problem sei. Zudem seien die nationalen Regelungsunterschiede auf dem Gebiet der Lebendorganspende nach wie vor derart groß, dass ein Zugang zu diesem Problem nicht allein auf nationaler, sondern auf europäischer Ebene zu suchen wäre. Basierend auf dem Aktionsplan und der Richtlinie wurde zunächst der Frage der Regelungsmöglichkeit der Lebendorganspende auf europäischer Ebene nachgegangen. Dabei zeigte sich, dass die EU nur über eine begrenzte Gemeinschaftskompetenz auf dem Gebiet der Lebendorganspende verfügt, da nach Art. 168 IV a) AEUV (ex Art. 152 EGV) die Kompetenz der EU auf die Vorgabe von Qualitäts- und Sicherheitsstandards begrenzt ist.

Da die EU in dem Aktionsplan und der Richtlinie impliziert hatte, dass ein Bedarf zur Festschreibung von Mindeststandards besteht, gleichzeitig aber die Gemeinschaftskompetenz beschränkt ist, sollte eruiert werden, inwiefern bereits Mindeststandards auf internationaler Ebene bestehen. Auf internationaler Ebene existierten im Zeitpunkt der Untersuchung bereits zwei Rechtsquellen, die sich thematisch mit der Lebendorganspende befasst hatten. Die Leitsätze der WHO, die Biomedizinkonvention sowie das Transplantationsprotokoll als Zusatzprotokoll der Biomedizinkonvention. Da diese nicht auf eine Gesetzgebungskompetenz angewiesen sind, sind diese weitaus detaillierter gefasst als die europäische Richtlinie. Diese internationalen Mindeststandards wurden sodann umfassend dargestellt, jedoch sehen sich beide Rechtsquellen letztlich dem Problem der mangelnden rechtlichen Verbindlichkeit ausgesetzt, da Rechtsakte der WHO lediglich als soft law qualifiziert werden können. Für die Biomedizinkonvention und das zugehörige Transplantationsprotokoll folgt dies aus dem Umstand, dass diese nur dann rechtsverbindlich sind, wenn sie unterzeichnet und ratifiziert wurden, was bislang bei nicht allen Ländern der Fall ist.

Im Anschluss an die vorhandenen internationalen Rechtsquellen, wurden die bereits existierenden nationalen Rechtsquellen zur Lebendorganspende exemplarisch anhand von Deutschland, England, Frankreich und Österreich untersucht, wobei der Harmonisierungsbedarf überhaupt erst anhand der nationalen Regelungen aufgezeigt werden sollte. Die Ausgangshypothese war, dass ein Harmonisierungsbedarf dann zwingend besteht, wenn die nationalen Regelungen zur Lebendorganspende derart divergieren, dass dies eine unterschiedliche Leistungsfähigkeit der nationalen Transplantationssysteme und schließlich auch strafanwendungsrechtliche Konsequenzen zur Folge hat. Zu diesem Zweck wurden die nationalen Regelungen anhand der Methodik des funktionalen Rechtsvergleichs, dem die Ähnlichkeitshypothese zugrundeliegt, miteinander verglichen. Im Zuge dieser Untersuchung konnte sich die Ähnlichkeitshypothese nur begrenzt bewahrheiten. So sind die nationalen Rege-lungsunterschiede nach wie vor insbesondere im Hinblick auf die in Deutschland und Frankreich existente strafbewehrte Spenderkreisbeschränkung so groß, dass England einem Modell „fehlender Restriktion“, Deutschland einem Modell „mittlerer Restriktion“ und Frankreich einem „starken Restriktionsmodell“ zuzuordnen sind. In Österreich fehlt es indes 
bereits an einer spezialgesetzlichen Regelung zur Lebendorganspende. Der Vergleich der nationalen Regelungen zeigte insgesamt, dass diese in weiten Teilen divergieren und dass dies reelle Auswirkungen auf das Organaufkommen hat.

Im Anschluss an den rechtsvergleichenden Teil der Untersuchung sollte zudem anhand des deutschen Strafanwendungsrechts und des dort existenten Gebots der identischen Tatnorm aufgezeigt werden, dass rechtliche Mindeststandards auch zur Vermeidung von Strafgefällen erforderlich sind.

Durch die Länderberichte zeigten sich jedoch auch in weiten Teilen Parallelen zu den internationalen Mindeststandards der Biomedizinkonvention und des Transplantationsprotokolls, was die Frage aufwarf, warum diese von den exemplarisch untersuchten Ländern nicht unterzeichnet und ratifiziert wurden. Dabei zeigte sich, dass der Grund hierfür ein typisches Problem von Rahmenkonventionen ist, die regelmäßig in dem Bestreben gefasst werden, möglichst viele Einzelthemen abzudecken, was immer die Gefahr einer mangelnden Konsensfähigkeit birgt. Damit zeigte der Vergleich mit der Biomedizinkonvention aber auch einen möglichen Lösungsansatz auf: Eine gesonderte Konvention zur Lebendspende würde einen praktikablen Ansatz zur Festschreibung von Mindeststandards im Mehr-Ebenen-System bieten:

Im Wesentlichen ließen sich drei Gründe ausmachen, warum eine gesonderte Europaratskonvention ein geeignetes Instrumentarium zur Festschreibung von Mindeststandards darstellen würde:

Zunächst ist eine Europaratskonvention im Gegensatz zu europäischen Rechtsakten nicht auf eine Gesetzgebungskompetenz angewiesen. Weiterhin sind Europaratskonventionen wenn sie denn unterzeichnet und ratifiziert sind - für die Unterzeichnerstaaten verbindlich und durch die inhaltliche Beschränkung der Konvention auf das Thema der Lebendorganspende wären konsensfähige Regelungen möglich, die sich auf die Hauptprobleme der Einwilligung in die Spende samt ihrer formellen Anforderungen und auf die Spenderkreisbeschränkung fokussieren könnten.

Aus diesem Grund wurde in der vorliegenden Untersuchung als Möglichkeit zur Festschreibung von Mindeststandards eine gesonderte Europaratskonvention vorgeschlagen, die in insgesamt 17 Artikeln dem Sachproblem der Lebendspende gerecht werden sollte, wobei auf eine harte Formulierung von Mindeststandards weitestgehend verzichtet wurde, um die Akzeptanz der Konvention nicht zu gefährden. Dennoch wird als Novum auf eine Spenderkreisbeschränkung verzichtet.

Zusammenfassend sollte anhand des exemplarischen Vergleichs der nationalen Regelungen zur Lebendorganspende verdeutlicht werden, dass nach wie vor ein Bedarf zur Festschreibung von einheitlichen Standards im Bereich der Lebendorganspende besteht, weswegen die nationalen Gesetzgeber auch ihre Distanz zu europäischen und internationalen Rechtsakten aufgeben sollten. Zugleich hat die Untersuchung jedoch auch verdeutlicht, dass der Festschreibung internationaler Standards eigene Probleme immanent sind, da die Ähnlichkeitshypothese keinen Absolutheitsanspruch hat: Denn die einzelnen Gesetze, die ein bestimmtes Sachproblem wie hier die Lebendspende lösen wollen, erfüllen innerhalb der nationalen Rechtsordnungen zum Teil unterschiedliche Funktionen, weswegen sich ein internationaler Konsens in der Vergangenheit immer schwierig gestaltet hat und 
sich wohl auch in Zukunft schwierig gestalten wird. Dennoch sollte die vorgeschlagene Konvention als probates Instrumentarium zur Festschreibung von einheitlichen Mindeststandards im Mehr-Ebenen-System und letztlich auch als Diskussionsbasis verstanden werden. 